\title{
A Gravitational Black Hole Algorithm for Autonomous UCAV Mission Planning in 3D Realistic Environments
}

\author{
A. A. Heidari \\ GIS Division, Geomatics Eng. Dep., College of \\ engineering, University of Tehran, Iran
}

\author{
R. A. Abbaspour \\ GIS Division, Geomatics Eng. Dep., College of \\ engineering, University of Tehran, Iran
}

\begin{abstract}
This article addresses a novel approach to 3D mission planning of UCAVs in constrained environments. To solve this NP-hard problem, black hole algorithm $(\mathrm{BH})$ is improved by considering stars gravities information. By modelling UCAV properties, aerospace constraints and DTM of environment, proposed mission planner based on black hole optimization algorithm is proposed. Also it provides a comparative study for efficiency evaluation of evolutionary 3D mission planners based on ACO, BA, DE, ES, GA, BH and PSO optimization algorithms. Then mission planning task of UCAV is performed. Simulations show the advantage of proposed gravitational $\mathrm{BH}$ mission planner.
\end{abstract}

\section{General Terms}

UCAV mission Planning, Artificial Intelligence, Autonomous Navigation, Mission Planning, Black Hole

\section{Keywords}

Unmanned combat aerial vehicle (UCAV), Flight simulation, 3D mission planning, Black hole optimization algorithm

\section{INTRODUCTION}

UCAV is from the family of unmanned aircrafts developed for performing reconnaissance missions. Long-range drones have an autopilot system for following predesigned waypoints and continue motion based on planned mission, when they are out of the control of station's communication range. Operational UAVs need human control, but operator tasks are based on UCAV level of autonomy. However, developments of intelligent unmanned flight systems have become a growing trend in many research areas. Trajectory planning is a vital task in autonomous control processes of UCAV navigation, which is responsible for producing optimal trajectories from the launching location to the landing station considering some known constraints in environment.

Many tasks should be applied to UCAV control systems for providing autonomous navigation. These steps maybe include scanning environment, DTM generation and mission planning. Mission planning is a complex requirement in the autonomous navigation. Its objective is to find an optimal flight path in proper time, to UAV be able to accomplish several mission tasks. Choosing efficient algorithms for solving mission planning problem is an influential task. Optimal mission planning relies on optimization technics, so it's usually solved offline. Use of UCAVs, which can fly autonomously in aerospace environments, is necessary in several innovative applications. Reliable safe navigation of UCAV in Complex missions has technical challenges and UCAV planning is an essential task. Aerospace applications of UCAVs require exact maneuvers and optimal decisions and robust mission planning algorithms. Complex space around UCAV flight trajectory makes the problem NP-hard.
Based on pervious literatures, trajectory planning problem was turned into novel hybrid methodologies based on ICA [1], neural network [2], fuzzy logic [3], ACO [4], PSO [5,6], GA [7] and the artificial potential field [8]. When we have large mission ranges in UCAV flight, trajectory planning will be a large scale constrained optimization process. General methods on 3D trajectory planning could be used to solve this NP-hard problem including $\mathrm{A}^{*}[9]$ and $\mathrm{D}^{*}$ and rapidly exploring Random Trees (RRT) [10] and other is potential fields, evolutionary techniques include PSO, GA, ACO and multiobjective evolutionary algorithms $[11,12]$. Every method has its own robustness in certain aspects that is related to the problem complexity.

The UCAV mission planning in realistic test fields is a wellknown optimization problem, so many algorithms have been designed to solve this multi-constrained problem, such as differential evolution [13], biogeography-based optimization $[14,15]$, genetic algorithm [16], ant colony algorithm [17] and its variant $[18,19]$, cuckoo search $[20,21]$, chaotic artificial bee colony [22], firefly algorithm [23,24], and intelligent water drops optimization [25], also algorithms such as immune GA (I-GA) [26], PSO [27], quantum-behaved PSO (Q-PSO) [28] and master-slave parallel vector-evaluated GA (MPV-GA) [29] have been applied.

Black hole algorithm is a swarm intelligence approach inspired from the black hole phenomenon [30]. In this paper, performance of this algorithm will be improved by considering stars gravities information. For this aim, kind of gravitational force among stars is defined and the movement of stars toward the black hole is adjusted based on computed universal gravity during the searching solution space. Then this algorithm is applied to the UCAV mission planning scheme.

The structure of the article is as follows. In Section 2 black hole Algorithm $(\mathrm{BH})$ is introduced and then Improved $\mathrm{BH}$ is proposed. Section 3 defines the UCAV mission planning problem and section 4 reflects the main results of UCAV mission planning in $3 \mathrm{D}$ aerospace. Conclusion is at the last section.

\section{GRAVITATIONAL BLACK HOLE ALGORITHM}

\subsection{Black Hole Phenomenon in Cosmology}

The theory of a black hole phenomenon is proposed based upon Einstein's general theory of relativity [31]. Black hole is expressed as existence of an infinite curvature in space-time [32]. Any close enough mass to the center of distortions, can't escape from gigantic gravitational field, including light [33]. Based on astrophysical explorations, there are many evidences that show supermassive stars with finished life cycle will be vanished in a form of black holes and make distortion in 
space-time texture [34]. Black hole has sphere-shaped boundary, or 'event horizon', that Inside of this horizon, it's impossible to escape from singularity of the central mass. Also the Schwarzschild geometry is the space-time geometry of empty space enclosing any spherical mass. The most remarkable result of this concept (based upon Figure 1) is named 'Schwarzschild radius' that is calculated according to Eq. (1):

$$
R=\frac{2 G M}{c^{2}} \approx 2.95 \frac{M}{M_{\text {sun }}} k m
$$

Where $\mathrm{G}$ is the gravitational constant, $\mathrm{M}$ shows the mass of black hole, and c donates the speed of light [35]. Curiously, Schwarzschild radius can be derived from Newtonian gravity and also corpuscular theory of light.

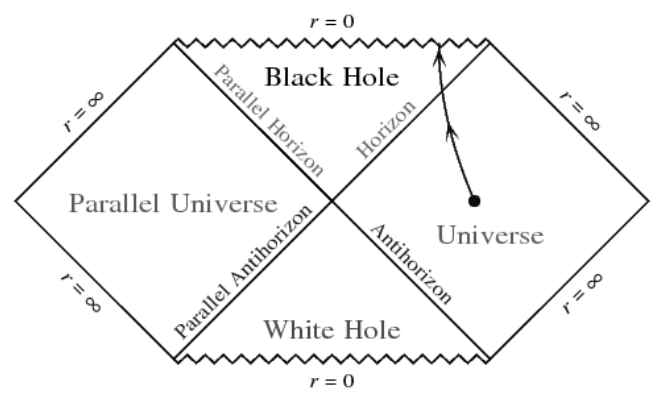

Fig. 1: Penrose diagram of Schwarzschild geometry [36]

Based upon no-hair theorem, black hole has three independent physical characteristics include mass, charge, and also angular momentum [37].

\subsection{Black Hole Optimization Algorithm}

This algorithm is a meta-heuristic algorithm introduced by Hatamlou [30] and it's based upon simulation of black hole formation process in cosmology for solving optimization tasks. At this swarm based optimization method, a population of solutions that are related to target problem should be scattered randomly in the search space. The population-based operators evolve the swarm members to find optimal solutions of problem. BH evolves the population by moving every star in the direction of the black hole, and replacing stars which are inside the horizon barrier by newly generated populations. Black holes are real candidates of the population. Then, all the candidates are assimilated toward the black hole. This operation is based on their Current position and a random value. The details of the $\mathrm{BH}$ optimization methodology are presented as follows:

After initializing step, fitness values of each star is computed and the best candidate among the population would be selected as black hole and the rest are chosen to be the normal stars in search space. Then the black hole start absorbing every star that is located in the neighborhood of central singularity point and consequently all the stars start moving towards the black hole center. In general, these motions frequently alternate the locations of stars individual toward the best fitness. The assimilation of stars caused by the black hole is formulated as Eq. (2):

$$
x_{i}(t+1)=x_{i}(t)+\operatorname{rand} \times\left(x_{B H}-x_{i}(t)\right) \quad i=1,2,3, \ldots, n
$$

where $x_{i}(t)$ and $x_{i}(t+1)$ represents the positions of $i$ th star at iterations $t$ and $t+1$, respectively. $\mathrm{x}_{B H}$ shows the location of black hole in search space, Rand is a random number in the interval $[0,1]$ and $\mathrm{N}$ is the number of stars. After moving step of the algorithm, if the cost value of a star individual was lower than the black hole, the position of them should be exchanged. During this process, there is a probability of crossing with event horizon. At the situation that a candidate is vanished into horizon, a new star is randomly born in the search space. The radius of Schwarzschild is calculated based upon the Eq. (3):

$$
\mathrm{R}=f_{\mathrm{BH}} / \sum_{i=1}^{\mathrm{N}} f_{i}
$$

where $f_{B H}$ donates the fitness of black hole, $f_{i}$ shows the fitness of $\mathrm{i}$ th star and $\mathrm{N}$ is the total number of stars.

\subsection{Proposed Black Hole Algorithm}

In general, the standard $\mathrm{BH}$ optimization method is adept at locating the promising area of global optimal point, but it is not relatively superior at exploiting target region. However, $\mathrm{BH}$ algorithm is weak to perform global search perfectly in the big problem spaces. In order to enhance the exploitation of $\mathrm{BH}$, a new operator performing local search is introduced to form a novel Gravitational black hole $(\mathrm{GBH})$ algorithm. For this aim, one can improve the absorption step of BH. In GBH, standard $\mathrm{BH}$ algorithm is applied to shrink the search area to a more promising region, and then absorption operator with good exploitation power is used to exploit the narrow region intensively to find better solutions with more convergence speed.

In this article, $\mathrm{BH}$ algorithm is improved based upon star gravitational field information as illustrated on Figure 2. For this purpose, kind of gravitational force between stars is defined and the movement operation of stars toward the black hole is simultaneously adjusted during the searching solution space. The difference between $\mathrm{GBH}$ and $\mathrm{BH}$ is that the absorption operator is applied to perform local search and fine-tune the original $\mathrm{BH}$ instead of random walks. Therefore, proposed GBH algorithm can fully overcome the lack of the exploitation of the $\mathrm{BH}$ and resolve the conflict among exploration and exploitation effectively. The detailed steps of GBH method is described as follows.

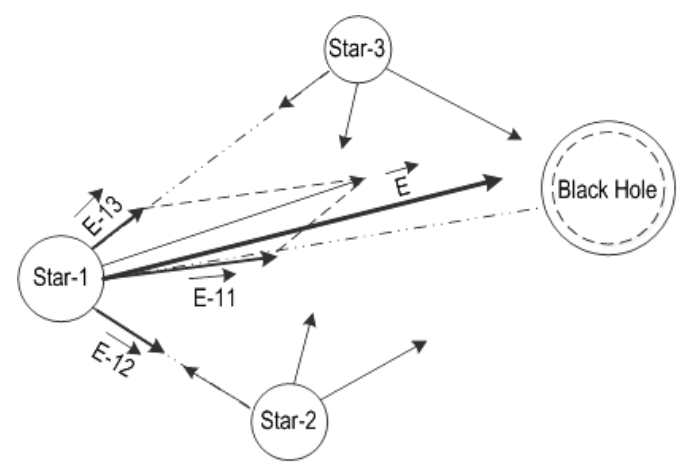

Fig. 2: Proposed movement operation based on gravitational fields

There is a swarm with $\mathrm{N}$ stars individuals. The position of the $\mathrm{i}$ th stars $\left(\mathrm{X}_{\mathrm{i}}\right)$ is defined by Eq. (4):

$$
X_{i}=\left(\text { star }_{i}, \ldots, \text { star }_{N}, \text { blackhole }_{d}\right)
$$

Where $s_{t a r}$ is the position of $i$ th star and blackhole $_{d}$ is the position of $d^{\text {th }}$ black hole, respectively. At a specific time' $\mathrm{t}$ ', absorption charge on star ' $i$ ' from star ' $j$ ' is as Eq. (5):

$$
E_{i j}^{d}(t)=\zeta\left(t_{0}\right) \frac{C_{p i}(t) \times C_{a j}(t)}{\left(D_{i j}(t)+\varepsilon\right)^{2}} \times\left(\frac{t_{0}}{t-t_{0}}\right)^{\alpha}
$$

where $C_{a j}$ is the absorption power of star $j, C_{p i}$ is the power related to star $i, \xi\left(\mathrm{t}_{0}\right)$ is initial absorption constant, $\alpha$ and $\varepsilon$ are values in $[0,1]$, and $D_{i j}(t)$ is defined as distance between two stars $i$ and $j$. to give a stochastic characteristic to $\mathrm{BH}$ algorithm, final force is randomly weighted sum of the forces of individuals as Eq. (6): 


$$
E_{i}^{d}(t)=\sum_{j=1 j \neq i}^{N} \operatorname{rand}_{j} E_{i j}^{d}(t)
$$

where $\operatorname{rand}_{j}$ is a value in $[0,1]$. Hence, the acceleration of the star $i$ at time $t$, and in direction $d^{\text {th }}$, is given by Eq. (7).

$$
a_{i}^{d}(t)=\frac{E_{i}^{d}(t)}{C_{i i}(t)}
$$

where $C_{i i}$ is the gravity force of $i^{\text {th }}$ star, also the next velocity of star is considered as follows. Therefore, position and its velocity are calculated based on Eq. (8) and Eq. (9).

$$
\begin{aligned}
& v_{i}^{d}(t+1)=\operatorname{rand}_{i} \times v_{i}^{d}(t)+a_{i}^{d}(t) \\
& \operatorname{star}_{i}(t+1)=\operatorname{star}_{i}(t)+v_{i}^{d}(t+1)
\end{aligned}
$$

The costs are updated by the Eq. (10), Eq. (11) and Eq. (12):

$$
\begin{aligned}
& C_{a i}=C_{p i}=C_{i i}=C_{i}, \quad i=1,2, \ldots, N \\
& c_{i}(t)=\frac{\operatorname{fit}_{i}(t)-\operatorname{weak}(t)}{\operatorname{strong}(t)-\operatorname{weak}(t)} \\
& C_{i}(t)=\frac{c_{i}(t)}{\sum_{j=1}^{N} c_{j}(t)}
\end{aligned}
$$

where $f i t_{i}(t)$ shows the fitness value of the star $i$ at time $t$, and weak $(t)$ and strong $(t)$ are based on Eq. 13 and Eq. 14:

$$
\begin{array}{ll}
\operatorname{strong}(t)=\min f i t_{j}(t), & j \in\{1, \ldots, N\} \\
\operatorname{weak}(t)=\max \text { fit }_{j}(t), & j \in\{1, \ldots, N\}
\end{array}
$$

\section{UCAV MISSION PLANNING PROBLEM}

Unmanned aerial systems should be capable to perform strategic missions with considering a variety of objectives. On the other hand, there are several conditions for an efficient mission planner including: optimality, completeness and complexity, which are related to vehicle motion dynamics. The extra dimensions of UCAV mission planning problem increase computational complexity for the evolutionary planner, because the design space is extended. Also Planner should be able to solve constrained optimization problems. In this article, designed environment of UCAV is a $3 \mathrm{D}$ realistic space that provide robust configuration of UCAV trajectories considering mission requirements (see Figure 3). In many of related works, Bezier curves have used for trajectory generations for computing smooth and feasible 3D routs for UAVs. Bezier curves are defined by $n$ control points include $P_{0}$ to $P_{n}$, where $n$ is called Bezier order .Polynomial form of the implemented Bezier curve is expressed as Eq. (15).

$$
\mathrm{B}(t)=\sum_{j=0}^{n} \frac{n !}{(n-j) !} t^{j} \sum_{i=0}^{j} \frac{(-1)^{i+j} \mathrm{P}_{i}}{i !(j-i) !}
$$

Regarding the evaluation of one candidate flight trajectories, Performance analysis of UCAV trajectory planning is mainly based on threat cost and fuel consumption, as in Eq. (16):

$$
C=k C_{\text {threat }}+(1-k) C_{\text {fuel }}
$$

where $\mathrm{k}$ should be in $[0,1]$, which provides certain flexibility for balancing between the threat degree and the fuel consumption indexes.

In this problem, we have Restriction conditions that must be satisfied during the optimization process. Based on Eq. (17) optimal trajectory of the UCAV must satisfy limitation constraints include UCAV turning radius $\left(\varphi_{S}\right)$, maximum flight height $\left(\mathrm{H}_{\max }\right)$, safe distance from terrain surface ( $S_{d}$ ) and escaping from flight prohibited zones (NFZs).

$$
x_{3}^{U C A V}-x_{3}^{D T M}<S_{d}, \varphi_{i, i+1}<\varphi_{S}, x_{3}^{\text {path }}<\mathrm{H}^{\max }
$$

where $\varphi_{\mathrm{i}, \mathrm{i}+1}$ is the angle between the extension of the line connecting Bezier points $i$ and $i+1, \varphi_{S}$ is the safe turning angle for controlling lateral and vertical accelerations. To avoid UCAV from terrain collision, $S_{\mathrm{d}}$ is a safe distance defined by control station team, $x_{3}^{\text {Path }}$ is the path curve coordinate, and $x_{3}{ }^{D T M}$ is the terrain point coordinate. $A^{L}$ is for limiting the peak height of UCAV.

It's proposed that UCAV flies with a constant speed, so the cost of the fuel consumption, $C_{\mathrm{F}}$, is computed based on the length of the UCAV path. $\mathrm{C}_{\mathrm{F}}$, in Eq. (18) is explained as the additional distance passed if the UCAV takes the alternate path compared to the original trajectory.

$$
\mathrm{C}_{\text {Fuel }}=\frac{5}{\mathrm{~L}_{0}} \sum_{i=1}^{n-1}\left|l\left(d_{i+1}\right)-l\left(d_{i}\right)\right|
$$

where $\mathrm{L}_{0}$ is computed by summing of all the line segments of primary trajectory and $\mathrm{l}\left(\mathrm{d}_{\mathrm{i}}\right)$ is the control point at $\mathrm{d} t h$ node on the traveled trajectory.

Modeling of threat sources in UCAV environment is the important task in mission planning. There are some threatening areas include radars, artilleries and missiles that is modeled in the shape of spheres. The probability of detection or crashing is proportional to inverse distance from the center of threat zones. It's demanded to find optimal trajectories in such a 3D aerospace at a proper time. To compute the threat avoidance cost, the distance from the UCAV to the threat zone should be resolved as expressed in Eq. (19).

$$
d\left(l\left(X_{i}\right), C_{t}\right)=\min \left[\operatorname{Dist}\left(1\left(\mathrm{X}_{i}, C_{t}\right)\right)-\mathrm{R}_{t}\right]
$$

where distance from UCAV position to the threat is $D_{t}$, and the radius of threat zone is donated by $\mathrm{R}_{\mathrm{t}}$. danger should be increased, when UCAV path is closer to the threat center. So the approximate formula is expressed in Eq. (20). The cost related to any threat is calculated for each trajectory portion. Then all segment cost values should be summed and scaled according to the number of danger zones. If the UCAV trajectory passed out of the threat zone, the segment cost values must all be 0 , and the total threat cost, $C_{t}$, will be 5 because of the predefined constant value.

$$
C_{\text {Threat }}=5+\left[\frac{1}{N_{t}}\right] \sum_{i=1}^{N} \sum_{j=1}^{N_{t}}\left[1-\frac{D_{t, j}^{4}}{R_{t}^{4}}\right]
$$

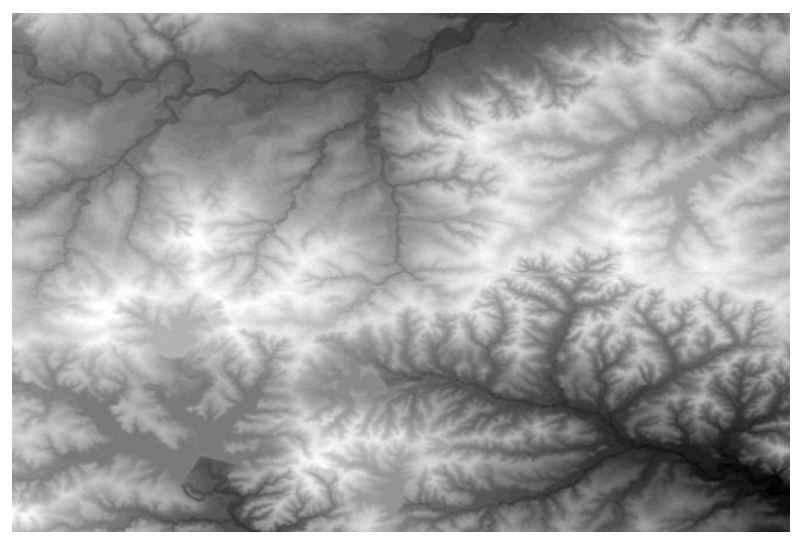

Fig. 3: Digital Terrain Model (DTM) as a realistic dataset for UCAV flight simulations. 


\section{EXPERIMENTAL RESULTS}

In this section, for evaluating the efficiency of proposed metaheuristic algorithm, various experiments are implemented. In order to achieve consistent evaluations of CPU times, Simulations of proposed GBH mission planner performed in the same PC and all the tests were under the same conditions. For performance analysis of ACO, BA, DE, ES, GA and PSO algorithms, parameters are selected based on Table1. To achieve to reliable results, Max-gen parameter of every algorithm is assigned to 100. For implementations MATLAB R2012a (7.14) environment used on a PC with $2.33 \mathrm{GHz}$ Intel Core 2 Duo and 4 GB of RAM memory. It is assumed that the mission region has $80 \mathrm{~km} \times 80 \mathrm{~km}$ size in a local reference system, UCAV Launching station is $(-40,40)$ and the UCAV Landing point is Located on $(40,-40)$. Flight altitude of UCAV defined within $(-20,20)$, and $\left(\varphi_{\mathrm{S}}, \mathrm{S}_{\mathrm{d}}\right)$ is $\left(55^{\circ}, 0.06\right)$.

Table 1. Parameter settings of meta-heuristic planners

\begin{tabular}{|c|l|}
\hline Code & \multicolumn{1}{|c|}{ Parameters } \\
\hline $\mathrm{ACO}[4]$ & $\begin{array}{l}\text { Initial pheromone value } \mathrm{s}_{0}=1 \mathrm{E}-5, \text { exploration } \\
\text { constant } \mathrm{q}_{0}=1, \text { local pheromone decay rate } \mathrm{q}_{\mathrm{l}}=0.4, \\
\text { global pheromone decay rate } \mathrm{q}_{\mathrm{g}}=0.8 \text {, pheromone } \\
\text { update constant } \mathrm{Q}=18, \text { visibility sensitivity } \mathrm{b}=6, \\
\text { pheromone sensitivity } \mathrm{s}=1\end{array}$ \\
\hline $\mathrm{BA}[38]$ & Loud $\mathrm{A}=0.85$, scaling e $=0.3$, pulse rate $\mathrm{r}=0.6$ \\
\hline $\mathrm{DE}[39]$ & crossover $\mathrm{CR}=0.5$, weighting factor $\mathrm{F}=0.95$ \\
\hline $\mathrm{ES}[40]$ & Off-springs $\mathrm{k}=20$, standard deviation $\mathrm{r}=1$ \\
\hline $\mathrm{GA}[7]$ & $\begin{array}{l}\text { Roulette wheel selection, single point crossover, } \\
\text { crossover probability of } 1, \text { mutation } 0.01\end{array}$ \\
\hline $\mathrm{PSO}[5]$ & $\begin{array}{l}\text { Inertial constant }=0.3, \text { social constant of swarm } \\
\text { interaction }=1, \text { cognitive constant }=1\end{array}$ \\
\hline
\end{tabular}

Comparative study about convergence speed of algorithms is performed on GA, PSO and BH and the results are presented on Figure 4. In this Figure, cost value of each mission planning simulation versus generations is illustrated. The average results for 100 correct runs have compared. During iterations of near 19 steps, cost value of GBH algorithm decreased rapidly. It's obvious that based on the results, GBH mission planner can find the optimal solution parameters very quickly. Based on Figure 4, apparently, GBH overtakes all other optimization methods. For this case, PSO and BH show a faster convergence rate initially, but GA and $\mathrm{BH}$ should be trapped into sub-optimal solutions as the procedure proceeds, especially GA, Although PSO performs the second best in computing process.

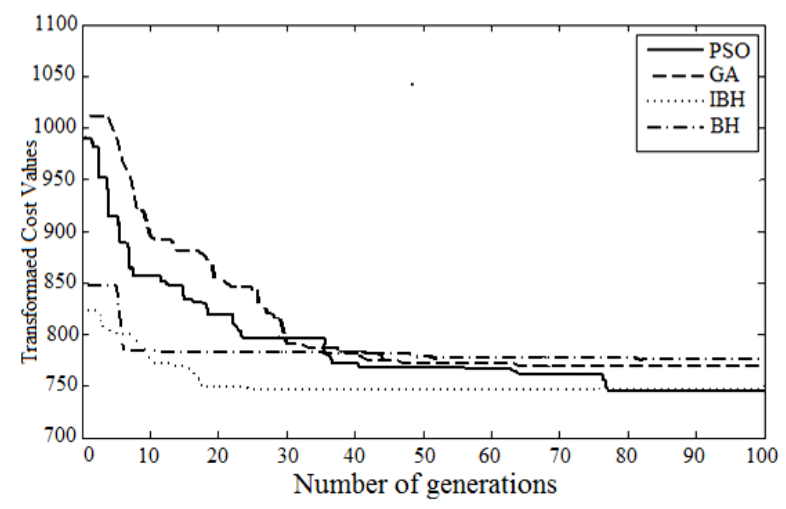

Fig 4: Plot of cost values of planes, related to PSO, GA, Improved BH (GBH) and BH.
Two scenarios are designed to evaluate the efficiency of metaheuristics algorithms in UCAV mission simulations. The differences between Scenario 1 and 2 are the 3D locations and numbers of the ground threats and the initial trajectory followed by UCAV. Each scenario cause challenges to the mission planner based on the location of threats. By selecting more threats in implemented scenario, UCAV must perform a complex maneuver to escape from threats and consequently the possibility of violating constraints will be increased. For results that are presented on Table 2, its subtracted 700 from the actual values, i.e., value of 57.351 is transformed value generated from the original number 757.351. The results of ACO, BA, DE, ES, GA, PSO, BH and GBH performance evaluations for UCAV mission planning problem are presented for tow different senarios in Table 2.

Table 2. Mean normalized optimization results and CPU performance time of UCAV mission planners

\begin{tabular}{|c|c|c|c|c|}
\hline \multirow{2}{*}{$\begin{array}{c}\text { Results } \\
\text { Method }\end{array}$} & \multicolumn{2}{|c|}{ Scenario-1 } & \multicolumn{2}{c|}{ Scenario-2 } \\
\cline { 2 - 5 } & Mean( \pm std) & CPU(s) & Mean( \pm std) & CPU(s) \\
\hline ACO[4] & $50.351 \pm 4.02$ & 34.5219 & $27.227 \pm 4.94$ & 16.4951 \\
\hline BA[38] & $47.589 \pm 4.28$ & 25.7715 & $19.448 \pm 2.01$ & 13.7111 \\
\hline DE[39] & $46.850 \pm 3.20$ & 24.4752 & $21.333 \pm 2.82$ & 14.8023 \\
\hline ES[40] & $48.271 \pm 4.57$ & 39.8450 & $25.736 \pm 7.11$ & 21.2793 \\
\hline GA[7] & $88.680 \pm 8.11$ & 45.1255 & $39.479 \pm 6.02$ & 26.5481 \\
\hline PSO[5] & $51.858 \pm 5.16$ & 24.3214 & $22.884 \pm 2.93$ & 18.7741 \\
\hline BH[30] & $59.712 \pm 6.35$ & 49.4670 & $29.582 \pm 5.15$ & 17.1120 \\
\hline GBH & $42.552 \pm 2.58$ & 23.1491 & $18.101 \pm 1.16$ & 12.9101 \\
\hline
\end{tabular}

Based on these results, GBH provides better results in comparision with other meta-heuristic methods. For example, in the case of senario-1, GBH in compare with ACO, BA, DE, ES, GA, PSO and $\mathrm{BH}$ provides results with less standard deviation and at least computional CPU time. In senario 2, solution quality of the results from BA and DE methods is especially superior in comparision with PSO, ES, ACO, BH and GA, respectively, but still GBH performs very well and is ranked one at computational time and solution qualities. In senario-1, Preformation of GBH is superior in comparison with other methods, while BA and DE should be ranked as second best in the term of std value among other algorithms, but fastest methods after GBH are PSO, DE and BA respectively. In senario-2, based upon $\mathrm{CPU}$ times, $\mathrm{GBH}$ significantly outperforms the BA, DE, ACO, BH, PSO, ES and GA respectively, when multiple executions are made. The results of BA, DE show that performances of these methods are almost similar. By comparing the results it is concluded that feasibility of the BA, PSO and DE is similar, GBH overtakes all other approaches and GA have the worst performances.

\section{CONCLUSION}

In this article, a novel approach is proposed for planning of 3D flight missions based on enhanced black hole algorithm. An efficient version of black hole methodology has been proposed for autonomous UCAV mission management, and an efficient improvement is applied to communicate among stars and black hole based upon relativity theory principles. This approach will improve global convergence speed of black hole method while robustness of original algorithm is maintained. Then, decision maker can discover any optimal mission plan by considering several limitations include threat sources and fuel consumptions. 
This work can enhance the autonomous UCAV's navigation, and guidance in 3D complex realistic missions. The proposed method based on BH optimization method has superior performances in compression with other well-known methods include ACO, BA, DE, ES, GA and PSO. Trajectories are computed using Bezier curve which can ascertain the generated trajectories for mission are smooth and flyable. Proposed method provides valid 3D trajectories with low computational complexity. The simulation results indicate that this novel GBH mission planner not only can produce safe 3D trajectories with more robustness, but also has higher convergence speed in comparison with other implemented algorithms.

\section{REFERENCES}

[1] E. Atashpaz-Gargari and C. Lucas, "Imperialist Competitive Algorithm: An Algorithm for Optimization Inspired by Imperialistic Competition". IEEE Congress on Evolutionary Computation, pp 4661-4667,2007.

[2] K. Althoefer, Neuro-fuzzy motion planning for robotic manipulators, Ph.D. thesis, King's College, London, 1997.

[3] Dong Z. N.?CZhang R. L.?CChen Z. J., et al. "Study on UAV Path Planning Approach Based on Fuzzy Virtual Force", Chinese Journal of Aeronautics, 23(3), 2010.

[4] C. Mou, W. Qing-xian, J. Chang-sheng, A modified ant optimization algorithm for path planning of UCAV, Applied Soft Computing 8 (4) ,pp.1712-1718,2007.

[5] M. Saska, M. Macas, L. Preucil, L. Lhotska ,Robot path planning using particle swarm optimization of Ferguson splines, in: Conf. Emerging Technologies and Factory Automation, IEEE, pp. 833-839, 2006.

[6] Foo, J.L., Knutzon, J., Kalivarapu, V., Oliver, J., and Winer, E.,"Path planning of unmanned aerial vehicles using B-Splines and particle swarm optimization", Journal of Aerospace Computing Information and Communication, 6(4), pp.271-290, 2009.

[7] S. Piao, B. Hong, "Path planning of robot using genetic annealing algorithm", in: Proc. of the 4th World Congress on Intelligent Control and Automation, vol. 1, pp. 493-495, 2002.

[8] M.G. Park, M.C. Lee, "Experimental evaluation of robot path planning by artificial potential field approach with simulated annealing", in: Proc. of the 41st SICE Annual Conf., vol. 4, pp. 2190-2195, 2006.

[9] Z. N. Dong, Z. J. Chen, R. Zhou, R. L. Zhang. "A hybrid approach of virtual force and $A^{*}$ search algorithm for UAV path re-planning". In Proceedings of the 6th IEEE International Conference on Industrial Electronics and Applications, IEEE, Beijing, China, pp.1140-1145, 2011.

[10] Melchior, N.A., Simmons, R.: "Particle RRT for path planning with uncertainty." In: Proceedings of the IEEE International Conference on Robotics and Automation, 2007

[11] S. Mittal and K. Deb, "Three-Dimensional Offline Path Planning for UAVs Using Multi objective Evolutionary Algorithms", Evolutionary Computation IEEE Conference, pp.1-6, 2007.

[12] Haibin Duan, Linzhi Huang,"Imperialist competitive algorithm optimized artificial neural networks for UCAV global path planning",Neurocomputing $125,166-171$, 2013.
[13] H. B. Duan,X.Y.Zhang, andC. F. Xu, "Bio-Inspired Computing", Science Press, Beijing, China, 2011.

[14] G. Wang, L. Guo, H. Duan, L. Liu, H. Wang, and M. Shao, "Path planning for uninhabited combat aerial vehicle using hybrid meta-heuristic DE/BBO algorithm," Advanced Science, Engineering and Medicine, vol. 4, no. 6, pp. 550-564, 2012.

[15] G. Wang, L. Guo, H. Duan, L. Liu, H. Wang, and M. Shao, "Hybridizing harmony search with biogeography based optimization for global numerical optimization," Journal of Computational and Theoretical Nanoscience.

[16] Y. V. Pehlivanoglu, “A new vibrational genetic algorithm enhanced with a Voronoi diagram for path planning of autonomous UAV," Aerospace Science and Technology, vol. 16, pp. 47-55, 2012.

[17] W. Ye, D. W. Ma, and H. D. Fan, “Algorithm for low altitude penetration aircraft path planning with improved ant colony algorithm," Chinese Journal of Aeronautics, vol. 18, no. 4, pp. 304-309, 2005.

[18] H. Duan, Y. Yu, X. Zhang, and S. Shao, "Threedimension path planning for UCAV using hybrid metaheuristic ACODE algorithm," Simulation Modelling Practice and Theory, vol. 18, no. 8, pp. 1104-1115, 2010.

[19] H. B. Duan, X. Y. Zhang, J. Wu, and G. J. Ma, "Maxmin adaptive ant colony optimization approach to multiUAVs coordinated trajectory replanning in dynamic and uncertain environments," Journal of Bionic Engineering, vol. 6, no. 2, pp.161-173, 2009.

[20] G. Wang, L. Guo, H. Duan, L. Liu, H. Wang, and B. Wang, "A hybrid meta-heuristic DE/CS algorithm for UCAV path planning," Journal of Information and Computational Science, vol. 5, no. 16, pp. 4811-4818, 2012.

[21] G. Wang, L. Guo, H. Duan, H. Wang, L. Liu, and M. Shao, "A hybrid meta-heuristic DE/CS algorithm for UCAV three dimension path planning," The Scientific World Journal, vol. 2012, Article ID 583973, 11 pages, 2012.

[22] C. Xu, H. Duan, and F. Liu, "Chaotic artificial bee colony approach to Uninhabited Combat Air Vehicle (UCAV) path planning," Aerospace Science and Technology, vol. 14, no. 8, pp. 535-541, 2010.

[23] G. Wang, L. Guo, H. Duan, L. Liu, and H. Wang, "A modified firefly algorithm for UCAV path planning," International Journal of Hybrid Information Technology, vol. 5, no. 3, pp. 123-144, 2012.

[24] A. H. Gandomi, X. S. Yang, S. Talatahari, and A. H. Alavi, "Firefly algorithm with chaos," Communications in Nonlinear Science and Numerical Simulation, vol. 18, no. 1, pp. 89-98, 2013.

[25] H. Duan, S. Liu, and J. Wu, "Novel intelligent water drops optimization approach to single UCAV smooth trajectory planning," Aerospace Science and Technology, vol. 13, no. 8, pp. 442-449, 2009.

[26] Z. Cheng, Y. Sun, and Y. Liu, "Path planning based on immune genetic algorithm for UAV," in Proceedings of the International Conference on Electric Information and Control Engineering (ICEICE '11), pp. 590-593, Wuhan, China, April 2011

[27] Y. Bao, X. Fu, and X. Gao, "Path planning for reconnaissance UAV based on particle swarm 
optimization," in Proceedings of the 2nd International Conference on Computational Intelligence and Natural Computing (CINC '10), pp. 28-32, Wuhan, China, September 2010.

[28] Y. Fu, M. Ding, and C. Zhou, "Phase angle-encoded and quantum-behaved particle swarm optimization applied to three-dimensional route planning for UAV," IEEE Transactions on Systems, Man, and Cybernetics A: Systems and Humans, vol. 42, no. 2, pp. 511-526, 2012.

[29] D. M. Pierre, N. Zakaria, and A. J. Pal, "Master-slave parallel vector-evaluated genetic algorithm for unmanned aerial vehicle's path planning," in Proceedings of the 11th International Conference on Hybrid Intelligent Systems (HIS '11), pp. 517-521,2011.

[30] Hatamlou, A., "Black hole: A new heuristic optimization approach for data clustering." Information Sciences 222, pp.175-184, 2013.

[31] Hawking, S.W, "Black hole explosions?" Nature 248, pp. $30-31,1974$.

[32] Hawking, S.W, "Breakdown of predictability in gravitational collapse" Commun. Math. Phys. vol.43, pp.199, 1975.

[33] Marco Angheben, Mario Nadalini, Luciano Vanzo and Sergio Zerbini, "Hawking radiation as tunneling for extremal and rotating black holes", Journal of High Energy Physics, vol.5, 2005.

[34] De-Jiang Qi, "Fermions Tunneling Mechanism for a New Class of Black Holes in EGB Gravity and ThreeDimensional Lifshitz Black Hole",Int. J. Theor. Phys, Vol.52, Issue 2, pp 345-353, 2013.

[35] Yang, S.Z., Jiang, Q.Q, "Research on Hawking Radiation as Tunneling from Schwarzshild-anti-de Sitter Black Hole", Int. J. Theor. Phys. Vol.46, Issue 8, pp. 2138 2145, 2007.

[36] Roger Penrose, "Conformal treatment of infinity" in Relativity, groups and topology ed. C. de Witt \& B. de Witt (Gordon and Breach, New York) p. 563-584, 1964; republished Gen. Rel. Grav. 43 901-922, 2011.

[37] Heusler, M., "Stationary Black Holes: Uniqueness and Beyond", Living Reviews in Relativity 1, 1998.

[38] A.H. Gandomi, X.-S. Yang, A.H. Alavi, S. Talatahari, "Bat algorithm for constrained optimization tasks", Neural Comput. Appl. 22 (6) pp.1239-1255, 2013.

[39] A.H. Gandomi, X.-S. Yang, S. Talatahari, S. Deb, "Coupled eagle strategy and differential evolution for unconstrained and constrained global optimization", Comput. Math. Appl. 63 (1) pp.191-200, 2012.

[40] T. Back, "Evolutionary Algorithms in Theory and Practice", Oxford University Press, 1996. 